\title{
Amphotericin B Deoxycholate
}

National Cancer Institute

\section{Source}

National Cancer Institute. Amphotericin B Deoxycholate. NCI Thesaurus. Code C62201.

The deoxycholate salt of amphotericin B, a polyene antifungal antibiotic produced by Streptomyces nodosus, with antifungal activity. Amphotericin B binds to ergosterol, an essential component of the fungal cell membrane. This results in depolarization of the cell membrane, alterations in cell membrane permeability, leakage of important intracellular components, and cell rupture. This agent may also induce oxidative damage in fungal cells and has been reported to stimulate host immune cells. 